\title{
Diophantine approximation on affine hyperplanes
}

by

\section{Anish Ghosh (Norwich)}

1. Introduction. In this paper we study Diophantine approximation on affine subspaces, extending and strengthening the main result in [G1]. Our approach uses the dynamics of flows on homogeneous spaces as developed in [KM] and [BKM].

We first recall the classical Khintchine-Groshev Theorem ([K], $\mathrm{Gr}, \mathrm{D}])$. The notation | | will be used to denote the absolute value of real numbers, as well as Lebesgue measure in $\mathbb{R}^{d}$, depending on context to make the usage clear, and \|| $\|$ will denote the supremum norm in $\mathbb{R}^{d}$,

$$
\|\mathbf{q}\|:=\max _{i}\left|q_{i}\right| .
$$

Let $\psi: \mathbb{R}_{+} \cup\{0\} \rightarrow \mathbb{R}_{+} \cup\{0\}$ be a nonincreasing function, and denote by $\mathcal{L}\left(\mathbb{R}^{n}, \psi\right)$ the set of $\mathbf{x} \in \mathbb{R}^{n}$ for which there exist infinitely many $\mathbf{q} \in \mathbb{Z}^{n}$ such that

$$
|p+\mathbf{x} \cdot \mathbf{q}|<\psi\left(\|\mathbf{q}\|^{n}\right)
$$

for some $p \in \mathbb{Z}$. The Khintchine-Groshev Theorem gives a precise measuretheoretic characterization of $\mathcal{L}\left(\mathbb{R}^{n}, \psi\right)$ in terms of the asymptotic properties of $\psi$ :

TheOREM 1.1. The set of $\psi$-approximable vectors $\mathcal{L}\left(\mathbb{R}^{n}, \psi\right)$ is null or co-null according as

$$
\sum_{k=1}^{\infty} \psi(k)
$$

converges or diverges.

It is significantly more difficult to obtain a similar characterization of the set $\mathcal{L}(\mathcal{S}, \psi)$ which is defined as in $(1.2)$ but with $\mathbf{x}$ restricted to lie on

2010 Mathematics Subject Classification: Primary 11J83; Secondary 11K60.

Key words and phrases: Diophantine approximation on manifolds, flows on homogeneous spaces. 
a proper submanifold $\mathcal{S}$ of $\mathbb{R}^{n}$. This is an instance of metric Diophantine approximation on manifolds ( $\mathrm{Sp}]$ ).

We recall briefly the notion of a nondegenerate manifold. Let $U$ be an open subset of $\mathbb{R}^{d}$ and let $\mathbf{f}: U \rightarrow \mathbb{R}^{n}$ be a differentiable map. Then $\mathbf{f}$ is said to be nondegenerate at $x_{0} \in U$ if the span of all the partial derivatives of $\mathbf{f}$ at $x_{0}$ up to some order equals $\mathbb{R}^{n}$, and nondegenerate if it is nondegenerate at almost every point of $U$. Finally, a smooth manifold $\mathcal{S}=\{\mathbf{f}(x): x \in U\}$ is called nondegenerate if $\mathbf{f}$ is. In [KM], D. Kleinbock and G. Margulis established a longstanding conjecture of V. G. Sprindzhuk by showing that almost every point on a nondegenerate manifold is not very well approximable, i.e. not $\psi_{\epsilon}$-approximable for $\psi_{\epsilon}(x)=x^{-n+\epsilon}$. Further in [BKM] and [BBKM], the convergence and divergence cases of the Khintchine-Groshev theorem were established for this class of manifolds. Less is known for degenerate manifolds, for example affine subspaces. Indeed, the theorem can be easily seen to fail unless some Diophantine condition is imposed on the parametrizing matrix of the subspace. In [Kl1] and [Kl2], extremality properties (i.e. the property that almost every point on a subspace is not very well approximable) of affine subspaces and their nondegenerate manifolds were studied. In [G1] and [G2], the convergence and divergence cases of the theorem were established for affine hyperplanes, i.e. codimension 1 affine subspaces, whose parametrizing matrices satisfy a certain Diophantine condition, and in [G3], the convergence case for affine subspaces and their nondegenerate submanifolds was investigated. Earlier, both cases of the Khintchine-Groshev Theorem were established in [BBDD] for lines passing through the origin.

1.1. Multiplicative and s-Diophantine approximation. We denote by $\operatorname{Mat}_{m, n}(\mathbb{R})$ the set of $(m, n)$-matrices with real entries. For an integer $n$ and a vector $\mathbf{q}=\left(q_{1}, \ldots, q_{n}\right) \in \mathbb{R}^{n}$, we set

$$
\Pi_{+}(\mathbf{q}):=\prod_{i=1}^{n} \max \left(\left|q_{i}\right|, 1\right) .
$$

The study of Diophantine approximation with the sup norm in (1.1) replaced by (1.4) is referred to as multiplicative Diophantine approximation. As noted in $[\mathrm{Bu}$, which is an excellent survey of the subject, this replacement is far from trivial and makes the analogous theorems much more difficult.

For $\mathbf{s} \in \mathbb{R}_{+}^{n}$ such that $\sum_{i=1}^{n} s_{i}=1$, we follow [BKM] in defining the s-quasinorm on $\mathbb{R}^{n}$ by

$$
\|\mathbf{x}\|_{\mathbf{s}}:=\max _{1 \leq i \leq n}\left|x_{i}\right|^{1 / s_{i}}
$$

For a submanifold $\mathcal{S}$ of $\mathbb{R}^{n}$, we can then define $\mathcal{L}(\mathcal{S}, \psi, \mathbf{s})$ and $\mathcal{L}(\mathcal{S}, \psi, \times)$ as in (1.2) but with the maximum norm $\|\mathbf{q}\|^{n}$ replaced by $\|\mathbf{q}\|_{\mathbf{s}}$ and $\Pi_{+}(\mathbf{q})$ respectively. We refer to these sets as $\psi$-s-approximable and $\psi$-multiplicatively 
approximable points respectively. Since $\psi$ is assumed to be nonincreasing, we have

$$
\mathcal{L}(\mathcal{S}, \psi, \mathbf{s}) \subseteq \mathcal{L}(\mathcal{S}, \psi, \times)
$$

and $\mathcal{L}(\mathcal{S}, \psi)=\mathcal{L}(\mathcal{S}, \psi,(1 / n, \ldots, 1 / n))$. The multiplicative version of the classical Khintchine-Groshev Theorem was established in Gal]. These questions have also been considered in the context of Diophantine approximation on manifolds. In $[\mathrm{KM}$, the stronger property that almost every point on a nondegenerate manifold is not very well multiplicatively approximable was established, and in [BKM], the multiplicative as well as $\mathbf{s}$ versions of the Khintchine-Groshev Theorem for nondegenerate manifolds were proved.

1.2. A Diophantine condition. We now set up some terminology to describe the Diophantine condition we will impose on the matrix parametrizing the hyperplane. For $v>0$ and $n \in \mathbb{Z}_{+}, \mathcal{W}_{v}(n, 1)$ is defined to be the set of all matrices $\mathfrak{a}=\left(a_{i}\right)_{0 \leq i \leq n-1} \in \operatorname{Mat}_{n, 1}(\mathbb{R})$ for which there are infinitely many $q \in \mathbb{Z}$ such that

$$
\max _{i}\left|p_{i}+a_{i} q\right|<|q|^{-v}
$$

for some $\mathbf{p} \in \mathbb{Z}^{n}$. We further define

$$
\begin{aligned}
& \mathcal{W}_{v}^{+}(n, 1)=\bigcup_{u>v} \mathcal{W}_{u}(n, 1), \\
& \mathcal{W}_{v}^{-}(n, 1)=\bigcap_{u<v} \mathcal{W}_{u}(n, 1) .
\end{aligned}
$$

It is an easy consequence of the definition that

$$
v_{1} \leq v_{2} \Rightarrow \mathcal{W}_{v_{2}}(n, 1) \subset \mathcal{W}_{v_{1}}(n, 1)
$$

and so

$$
\mathcal{W}_{v}^{+}(n, 1) \subseteq \mathcal{W}_{v}(n, 1) \subseteq \mathcal{W}_{v}^{-}(n, 1)
$$

If $v>1 / n$, then as a consequence of the Borel-Cantelli lemma, the Lebesgue measure of $\mathcal{W}_{v}(n, 1)$ is zero. In particular, $\mathcal{W}_{1 / n}^{+}(n, 1)$ has measure zero. This is dual to the situation in (1.2) and related to it by Khintchine's transference principle (cf. $\left[\mathrm{Sp}\right.$ ). For a vector $\mathbf{x}=\left(x_{1}, \ldots, x_{n}\right) \in \mathbb{R}^{n}$, we will denote the vector $\left(1, x_{1}, \ldots, x_{n}\right) \in \mathbb{R}^{n+1}$ by $\tilde{\mathbf{x}}$. Let $\mathfrak{a}=\left(a_{0}, \ldots, a_{n-1}\right)^{T} \in \operatorname{Mat}_{n, 1}(\mathbb{R})$ and let $\mathscr{H}$ be the affine hyperplane defined by $\mathfrak{a}$, i.e.

$$
\mathscr{H}=\left\{(\mathbf{x}, \tilde{\mathbf{x}} \mathfrak{a}): \mathbf{x} \in \mathbb{R}^{n-1}\right\} .
$$

In [Kl1], the condition $\mathfrak{a} \notin \mathcal{W}_{n}^{+}(n, 1)$ was shown to be both necessary and sufficient for $\mathscr{H}$ to be extremal, and similarly the condition $\mathfrak{a} \notin \mathcal{W}_{r}^{+}(n, 1)$ for strong extremality, i.e. the property that almost every point is not very 
well multiplicatively approximable $\left({ }^{1}\right)$. Here

$$
r=r(\mathfrak{a})=\#\left\{1 \leq i \leq n-1: a_{i} \neq 0\right\} .
$$

In G1, it was shown that the condition

$$
\mathfrak{a} \notin \mathcal{W}_{n}^{-}(n, 1)
$$

is sufficient to prove the convergence case of the Khintchine-Groshev Theorem for $\mathscr{H}$. Our first result improves this result to s-norms.

Theorem 1.2. Suppose a satisfies 1.10. Then for every $\mathbf{s} \in \mathbb{R}_{+}^{n}$ with $\sum_{i=1}^{n} s_{i}=1, \mathcal{L}(\mathscr{H}, \psi, \mathbf{s})$ is a null set whenever $\sum_{k=1}^{\infty} \psi(k)$ converges.

Our next result treats the multiplicative situation.

Theorem 1.3. Assume that $r(\mathfrak{a})=n-1$ and that $\mathfrak{a}$ satisfies (1.10). Then $|\mathcal{L}(\mathscr{H}, \psi, \times)|=0$ whenever $\sum_{k=1}^{\infty}(\log k)^{n-1} \psi(k)$ converges.

We will mostly concentrate on proving Theorem 1.3 , which is the main result of this paper. The last section will be devoted to explaining the changes in the proof which will yield Theorem 1.2 .

2. First steps. Since being $\psi$-approximable is a property which is invariant under translation by integers, we may restrict $\mathbf{x}$ to lie in a fixed ball $\mathscr{B} \subset \mathbb{R}^{n-1}$. Let

$$
\mathcal{L}(\mathscr{B}, \psi, \mathbf{q})=\left\{\mathbf{x} \in \mathscr{B}:|p+(\mathbf{x}, \tilde{\mathbf{x}} \mathfrak{a}) \cdot \mathbf{q}|<\psi\left(\Pi_{+}(\mathbf{q})\right) \text { for some } p \in \mathbb{Z}\right\}
$$

and

$\mathcal{L}_{>}(\mathscr{B}, \psi, \mathbf{q})=\left\{\mathbf{x} \in \mathscr{B}: \exists p \in \mathbb{Z}\right.$ such that $|p+\mathbf{q} \cdot(\mathbf{x}, \tilde{\mathbf{x}} \mathfrak{a})|<\psi\left(\Pi_{+}(\mathbf{q})\right) ;$

$$
\left.\left|q_{i}+a_{i} q_{n}\right|>1 \text { for some } 1 \leq i \leq n-1\right\}
$$

and define $\mathcal{L}_{\leq}(\mathscr{B}, \psi, \mathbf{q})=\mathcal{L}(\mathscr{B}, \psi, \mathbf{q}) \backslash \mathcal{L}_{>}(\mathscr{B}, \psi, \mathbf{q})$. Our plan is to apply the convergence case of the Borel-Cantelli lemma; we will begin by proving that

$$
\sum_{\mathbf{q} \in \mathbb{Z}^{n}}\left|\mathcal{L}_{>}(\mathscr{B}, \psi, \mathbf{q})\right|<\infty .
$$

It follows from Lemma 3.2 in G1] that

$$
\left|\mathcal{L}_{>}(\mathscr{B}, \psi, \mathbf{q})\right| \leq C(n, \mathscr{B}) \psi\left(\Pi_{+}(\mathbf{q})\right) .
$$

We therefore have

$\left({ }^{1}\right)$ In fact stronger results in [Kl1 include conditions for extremality and strong extremality for nondegenerate submanifolds of $\mathscr{H}$. Khintchine type theorems for nondegenerate submanifolds of affine subspaces will be established in a forthcoming work. 


$$
\begin{aligned}
\sum_{\mathbf{q} \in \mathbb{Z}^{n}}\left|\mathcal{L}_{>}(\mathscr{B}, \psi, \mathbf{q})\right| & \leq C(n, \mathscr{B}) \sum_{\mathbf{q} \in \mathbb{Z}^{n}} \psi\left(\Pi_{+}(\mathbf{q})\right) \\
& \asymp \sum_{k=1}^{\infty}(\log k)^{n-1} \psi(k)<\infty,
\end{aligned}
$$

which establishes 2.2 .

3. A dynamical reformulation. The fact that $\psi$ is nonincreasing coupled with the requirement that $\sum_{k=1}^{\infty}(\log k)^{n-1} \psi(k)<\infty$ means that for all $\mathbf{q} \in \mathbb{Z}_{+}^{n}$ with large enough norm,

$$
\psi\left(\Pi_{+}(\mathbf{q})\right) \leq\left(\prod_{i=1}^{n}\left|q_{i}\right|\right)^{-1} .
$$

For $\mathbf{t}=\left(t_{1}, \ldots, t_{n}\right) \in \mathbb{Z}_{+}^{n}$, we set $t:=\sum_{i=1}^{n} t_{i}$. Let

$\mathcal{L}_{\leq}(\mathscr{B}, \mathbf{t})=\left\{\mathbf{x} \in \mathscr{B}: \exists p \in \mathbb{Z}, \mathbf{q} \in \mathbb{Z}^{n} \backslash\{0\}\right.$ such that $|p+\mathbf{q} \cdot(\mathbf{x}, \tilde{\mathbf{x}} \mathfrak{a})|<2^{-t} ;$

$$
\left.\left|q_{i}+a_{i} q_{n}\right| \leq 1 \text { for every } 1 \leq i \leq n-1 ;\left|q_{i}\right|<2^{t_{i}}\right\} .
$$

In view of (3.1), we have

$$
\bigcup_{\mathbf{q} \in \mathbb{Z}^{n}, 2^{t_{i}-1} \leq\left|q_{i}\right|<2^{t_{i}}} \mathcal{L}_{\leq}(\mathscr{B}, \psi, \mathbf{q}) \subset \mathcal{L}_{\leq}(\mathscr{B}, \mathbf{t}) .
$$

Let $\mathfrak{a}^{\prime} \in \operatorname{Mat}_{n-1,1}(\mathbb{R})$ denote the matrix $\left(a_{1}, \ldots, a_{n-1}\right)^{T}$, set

$$
\mathfrak{u}_{\mathbf{x}}=\left(\begin{array}{cccc}
1 & 0 & \mathbf{x} & \tilde{\mathbf{x}} \mathfrak{a} \\
0 & \mathrm{I}_{n-1} & \mathrm{I}_{n-1} & \mathfrak{a}^{\prime} \\
0 & 0 & \mathrm{I}_{n-1} & 0 \\
0 & 0 & 0 & 1
\end{array}\right),
$$

and for $\beta>0$, let

$$
\mathfrak{g}_{\beta, \mathbf{t}}=\operatorname{diag}\left(\frac{2^{-\beta t}}{2^{-t}}, 2^{-\beta t}, \ldots, 2^{-\beta t}, \frac{2^{-\beta t}}{2^{t_{1}}}, \ldots, \frac{2^{-\beta t}}{2^{t_{n}}}\right) .
$$

We also denote by $\Lambda$ the subgroup of $\mathbb{Z}^{n+d+1}$ defined by

$$
\Lambda=\left\{\left(\begin{array}{c}
p \\
0 \\
\vdots \\
0 \\
\mathbf{q}^{t}
\end{array}\right): p \in \mathbb{Z}, \mathbf{q} \in \mathbb{Z}^{n}\right\} .
$$

It is easy to see that

$$
\mathcal{L}_{\leq}(\mathscr{B}, \mathbf{t}) \subseteq\left\{\mathbf{x} \in \mathscr{B}:\left\|\mathfrak{g}_{\beta, \mathbf{t}} \mathfrak{u}_{\mathbf{x}} \mathbf{l}\right\|<2^{-\beta t} \text { for some } \mathbf{l} \in \Lambda \backslash\{0\}\right\} .
$$


Note that using induction on dimension as in [BKM], we can restrict ourselves to integer vectors $\mathbf{q}$ all of whose coordinates are nonzero. Therefore in view of 2.2 and 3.2 , it is enough to show the following.

Theorem 3.1. Assume that $\mathfrak{a}$ satisfies the conditions in Theorem 1.3 . Then for every $0<\beta<1 / n^{2}$, there exist $T(\beta)>0$ and $0<\gamma<\beta$ such that for any $\mathbf{t} \in \mathbb{Z}_{+}^{n}$ with $t \geq T(\beta)$,

$$
\mid\left\{\mathbf{x} \in \mathscr{B}:\left\|\mathfrak{g}_{\beta, t} \mathfrak{u}_{\mathbf{x}} \mathbf{l}\right\|<2^{-\beta t} \text { for some } \mathbf{l} \in \Lambda \backslash\{0\}\right\}\left|\leq C^{\prime} 2^{-(\beta-\gamma) t}\right| \mathscr{B} \mid,
$$
where $C^{\prime}$ is a constant independent of $\beta$ and $\gamma$.

The rest of the paper will be devoted to this proof.

3.1. Good functions. We define and state some basic properties of good functions, a concept introduced in [KM] which will play a crucial role in the next section.

Definition 3.2. Let $C$ and $\alpha$ be positive numbers and $V$ be a subset of $\mathbb{R}^{d}$. A function $f: V \rightarrow \mathbb{R}$ is said to be $(C, \alpha)$-good on $V$ if for any open ball $B \subseteq V$, and for any $\epsilon>0$, one has

$$
\left|\left\{\mathbf{x} \in B:|f(\mathbf{x})|<\epsilon \sup _{\mathbf{x} \in B}|f(\mathbf{x})|\right\}\right| \leq C \epsilon^{\alpha}|B| .
$$

Some easy properties of $(C, \alpha)$-good functions are:

- $f$ is $(C, \alpha)$-good on $V \Rightarrow$ so is $\lambda f$ for all $\lambda \in \mathbb{R}$.

- $f_{i}, i \in I$, are $(C, \alpha)$-good $\Rightarrow$ so is $\sup _{i \in I}\left|f_{i}\right|$.

It turns out that polynomials are prototypical examples of $(C, \alpha)$-good functions (see for instance $[\mathrm{KM}]$ ). For our purposes, the following elementary result ([G1, Lemma 4.2]) will suffice:

Lemma 3.3. Let $V \subset \mathbb{R}^{d}$, and $f$ be a (continuous) linear function on $V$. Then $f$ is $\left(C_{d}, 1\right)$-good on $V$, where $C_{d}=2^{d+2} / v_{d}$, and $v_{d}$ is the volume of the unit ball in $\mathbb{R}^{d}$.

4. A quantitative nondivergence estimate. For $k \geq 2$ let $\Lambda$ be a discrete subgroup of $\mathbb{R}^{k}$. A subgroup $\Gamma$ of $\Lambda$ is said to be primitive in $\Lambda$ if $\Gamma=$ $\Gamma_{\mathbb{R}} \cap \Lambda$, where $\Gamma_{\mathbb{R}}$ denotes the minimal linear subspace of $\mathbb{R}^{l}$ containing $\Gamma$. Let $\mathscr{P}(\Lambda)$ be the set of all nonzero primitive subgroups of $\Lambda$. Let $\Gamma \subseteq \mathbb{R}^{l}$ be one such subgroup. If $k=\operatorname{dim}\left(\Gamma_{\mathbb{R}}\right)$, for any basis $\mathbf{v}_{1}, \ldots, \mathbf{v}_{k}$ of $\Gamma$ the vector $\mathbf{v}_{1} \wedge \cdots \wedge \mathbf{v}_{k} \in \wedge^{k}\left(\mathbb{R}^{l}\right)$ is (up to a sign) independent of the basis. It is therefore natural to define $\|\Gamma\|=\|\mathbf{w}\|$ where $\mathbf{w}=\mathbf{v}_{1} \wedge \cdots \wedge \mathbf{v}_{k}$ is said to represent $\Gamma$. This norm can be extended as follows: For $I=\left(i_{1}, \ldots, i_{j}\right) \subset\{1, \ldots, l\}$ with 
$i_{1}<\cdots<i_{j}$, let

$$
e_{I}=e_{i_{1}} \wedge \cdots \wedge e_{i_{j}} \in \bigwedge^{j}\left(\mathbb{R}^{l}\right)
$$

Then for

$$
\mathbf{w}=\sum_{I \subset\{1, \ldots, l\}} \mathbf{w}_{I} e_{I}
$$

we define $\|\mathbf{w}\|=\max _{I \subset\{1, \ldots, k\}}\left|\mathbf{w}_{I}\right|$ with the additional convention that $e_{\emptyset}=1$. We now state a general measure estimate from [BKM], which is a refinement of an earlier estimate in $\left[\mathrm{KM}\right.$. Set $N_{d}$ to be the Besicovitch constant for $\mathbb{R}^{d}$.

TheOREM 4.1. For arbitrary $l \geq 2$, let $\Lambda$ be a discrete subgroup of $\mathbb{R}^{l}$ with $\operatorname{rank}(\Lambda)=k$. Further, let a ball $B\left(\mathbf{x}_{0}, r_{0}\right) \subset \mathbb{R}^{d}$ and a continuous map $H: \tilde{B} \rightarrow \mathrm{GL}\left(\mathbb{R}^{l}\right)$ be given, where $\tilde{B}$ stands for $B\left(\mathbf{x}_{0}, 3^{k} r_{0}\right)$. Take $C, \alpha>0$ and $0<\rho \leq 1 / k$, and let $\|\cdot\|$ be the supremum norm on $\Lambda\left(\mathbb{R}^{l}\right)$ (as defined before). Assume that for any $\Gamma \in \mathscr{P}(\Lambda)$,

(KM1) the function $\mathbf{x} \mapsto\|H(\mathbf{x}) \Gamma\|$ is $(C, \alpha)$-good on $\tilde{B}$,

(KM2) there exists $\mathbf{x} \in B$ such that $\|H(\mathbf{x}) \Gamma\| \geq \rho$.

Also assume that

(KM3) for all $\mathbf{x} \in \tilde{B}, \#\{\Gamma \in \mathscr{P}(\Lambda):\|H(\mathbf{x}) \Gamma\|<\rho\}<\infty$.

Then for every positive $\epsilon \leq \rho$ one has

$$
\begin{aligned}
\mid\{\mathbf{x} \in B:\|H(\mathbf{x}) \lambda\|<\epsilon \text { for some } \lambda \in \Lambda \backslash & \{0\}\} \mid \\
& <k\left(3^{d} N_{d}\right)^{k} C(\epsilon / \rho)^{\alpha}|B| .
\end{aligned}
$$

We now turn to the

Proof of Theorem 3.1. We take $\mathscr{B}$ which was fixed before and

$$
H: \tilde{\mathscr{B}} \rightarrow \mathrm{GL}(n+d+1, \mathbb{R}), \quad H(\mathbf{x})=\mathfrak{g}_{\beta, \mathbf{t}} \mathfrak{u}_{\mathbf{x}},
$$

and $\epsilon=\epsilon(t)=2^{-\beta t}$ for some $\beta \in \mathbb{R}_{+}$. From Lemma 3.3, it follows that (KM1) is satisfied with $(C, \alpha)=\left(C_{n-1}, 1\right)$. As for (KM3), this follows from the discreteness of $\bigwedge(\Gamma)$ in $\bigwedge\left(\mathbb{R}^{n+1}\right)$. In the next section, we will show

Proposition 4.2. For every $0<\beta<1 / n^{2}$, there exist $T(\beta)>0$ and $0<\gamma<\beta$ such that for any $\mathbf{t} \in \mathbb{Z}_{+}^{n}$ with $t \geq T(\beta)$,

$$
\inf _{\Gamma \in \mathscr{P}(\Lambda)} \sup _{\mathbf{x} \in \mathscr{B}}\|H(\mathbf{x}) \Gamma\| \geq 2^{-\gamma t} .
$$

We now take $\rho=\rho(t)=2^{-\gamma t}$ and apply Theorem 4.1 to get

$$
\mid\left\{\mathbf{x} \in \mathscr{B}:\|H(\mathbf{x}) \lambda\|<2^{-\beta t} \text { for some } \lambda \in \Lambda \backslash\{0\}\right\}\left|<C^{\prime} 2^{-(\beta-\gamma) t}\right| \mathscr{B} \mid,
$$

where $C^{\prime}=(n+1)\left(3^{n-1} N_{n-1}\right)^{n+1} C_{n-1}$, which establishes Theorem 3.1 . 
5. Lower bounds. In this section, we prove Proposition 4.2 , i.e. we obtain lower bounds for $\|H(\mathbf{x}) \Gamma\|$. Let $V=\operatorname{span}\left(\mathbf{e}_{0}, \mathbf{e}_{* 1}, \ldots, \mathbf{e}_{* d}, \mathbf{e}_{1}, \ldots, \mathbf{e}_{n}\right)$, $V^{*}=\operatorname{span}\left(\mathbf{e}_{* 1}, \ldots, \mathbf{e}_{* d}\right)$ and $V^{\prime}=\operatorname{span}\left(\mathbf{e}_{0}, \mathbf{e}_{1}, \ldots, \mathbf{e}_{n}\right)$. We employ the following strategy. For each $1 \leq k \leq n+1$, we take a vector $\mathbf{w} \in \bigwedge^{k}\left(V^{\prime}\right)$ (which represents a primitive subgroup), and explicitly compute $\mathfrak{g}_{\beta, \mathbf{t}} \mathfrak{u}_{\mathbf{x}} \mathbf{w}$. Let $\mathbf{w} \in \bigwedge^{k}\left(V^{\prime}\right)$ for some $k \geq 1$. Then

$$
\mathbf{w}=\sum_{I, \# I=k} w_{I} \mathbf{e}_{I}
$$

We first describe the action of $\mathfrak{u}_{\mathrm{x}}$ on the basis vectors of $V$ :

$$
\begin{aligned}
\mathfrak{u}_{\mathbf{x}} \mathbf{e}_{0} & =\mathbf{e}_{0}, \\
\mathfrak{u}_{\mathbf{x}} \mathbf{e}_{* i} & =\mathbf{e}_{* i}, \\
\mathfrak{u}_{\mathbf{x}} \mathbf{e}_{i} & =x_{i} \mathbf{e}_{0}+\mathbf{e}_{* i}+\mathbf{e}_{i} \quad \text { for } 1 \leq i \leq n-1, \\
\mathfrak{u}_{\mathbf{x}} \mathbf{e}_{n} & =\left(a_{0}+\sum_{i=1}^{n-1} a_{i} x_{i}\right)+\sum_{i=1}^{n-1} a_{i} \mathbf{e}_{* i}+\mathbf{e}_{n} .
\end{aligned}
$$

5.1. The rank 1 case. We first deal with the case when $\Gamma \in \mathscr{P}(\Lambda)$ is of rank 1. We isolate this case for one important reason: the existence of a lower bound for $\|H(\mathbf{x}) \Gamma\|$ depends quite sensitively on the Diophantine properties of $\mathfrak{a}$. This is in contrast to the higher rank case where different considerations play a role. Accordingly, we take a vector $\mathbf{w} \in \bigwedge^{1}\left(V^{\prime}\right)$,

$$
\mathbf{w}=p_{0} \mathbf{e}_{0}+q_{1} \mathbf{e}_{1}+\cdots+q_{n} \mathbf{e}_{n} .
$$

Then

$$
\begin{aligned}
\mathfrak{g}_{\beta, \mathbf{t}} \mathfrak{u}_{\mathbf{x}} \mathbf{w}= & \frac{2^{-\beta t}}{2^{-t}}\left(p_{0}+\sum_{i=1}^{n} q_{i} x_{i}\right) \mathbf{e}_{0}+2^{-\beta t}\left(\sum_{i=1}^{n-1}\left(q_{i}+q_{n} a_{i}\right) \mathbf{e}_{* i}\right) \\
& +\sum_{i=1}^{n}\left(\frac{2^{-\beta t}}{2^{t_{i}}} q_{i} \mathbf{e}_{i}\right) .
\end{aligned}
$$

We now discuss briefly and informally the process of bounding $\left\|\mathfrak{g}_{\beta, \mathbf{t}} \mathfrak{u}_{\mathbf{x}} \mathbf{w}\right\|$ from below. The action of $\mathfrak{g}_{\beta, \mathbf{t}}$ expands $\mathbf{e}_{0}$ and contracts the other vectors. It is therefore our hope that the coefficient of $\mathbf{e}_{0}$ does not become very small on $\mathscr{B}$. This coefficient is

$$
\left(p_{0}+q_{n} a_{0}\right)+\sum_{i=1}^{n-1} x_{i}\left(q_{i}+q_{n} a_{i}\right) .
$$

It is here that the Diophantine property, i.e. the fact that $\mathfrak{a}$ is far from rational, plays a crucial part in ensuring that (5.4) is typically not very small (where "small" is measured by a power of $\left(q_{1}, \ldots, q_{n}\right)$ as in the Diophantine condition assumed in Theorem 1.3). In the event that the $\left|q_{i}+q_{n} a_{i}\right|$ 
do become small, the terms $\left(2^{-\beta t} / 2^{t_{i}}\right) q_{i} \mathbf{e}_{i}$ in 5.3 are used to ensure that $\sup _{\mathbf{x} \in B}\left\|\mathfrak{g}_{\beta, \mathbf{t}} \mathfrak{u}_{\mathbf{x}} \mathbf{w}\right\|$ is bounded below. Before making this argument rigorous, we rephrase the conditions imposed in Theorem 1.3 to a slightly more convenient form. For a vector $\mathbf{p}=\left(p_{0}, p_{1}, \ldots, p_{n-1}\right)$, we set $\mathbf{p}^{\prime}:=\left(p_{1}, \ldots, p_{n-1}\right)$. Then we have

Lemma 5.1. Suppose $r(\mathfrak{a})=n-1$ where $r(\mathfrak{a})$ is defined as in (1.9). Then $\mathfrak{a}$ satisfies 1.10 if and only if there exists $\delta>0$ such that

$$
\|\mathbf{p}+\mathfrak{a} q\|>\Pi_{+}\left(\mathbf{p}^{\prime}, q\right)^{(-n+\delta) / n}
$$

for every $\mathbf{p} \in \mathbb{Z}^{n}$ and all but finitely many $q \in \mathbb{Z}$.

Proof. Assume first that $\mathfrak{a}$ as above does not satisfy (5.5). Then, since each $a_{i}$ is nonzero, it follows that for each $i,\left|p_{i}\right| \asymp|q|$, i.e. their ratios can be bounded from both sides. Consequently, $\Pi_{+}\left(\mathbf{p}^{\prime}, q\right)^{(-n+\delta) / n}$ can be replaced with $|q|^{(-n+\delta)}$ by at most changing $\delta$ slightly in the latter expression, and we have thus shown that $\mathfrak{a} \in \mathcal{W}_{n}^{-}(n, 1)$. The converse is identical and is omitted.

We now set $\mathbf{p}=\left(p_{0}, q_{1}, \ldots, q_{n-1}\right), \mathbf{p}^{\prime}=\left(q_{1}, \ldots, q_{n-1}\right)$ and $q=q_{n}$ and prove

Lemma 5.2. Assume that there exists $\beta>0$ such that for every $0<\gamma$ $<\beta$, there exists an unbounded set of $\mathbf{t} \in \mathbb{R}_{+}^{n}$ such that:

(1) $2^{-\beta t} 2^{t}\|\mathbf{p}+\mathfrak{a} q\| \leq 2^{-\gamma t}, 2^{-\beta t} 2^{-t_{i}}\left|q_{i}\right| \leq 2^{-\gamma t}$ for $1 \leq i \leq n-1$.

(2) $2^{-\beta t} 2^{-t_{n}}|q| \leq 2^{-\gamma t}$.

Then for every $v<n$, there exists $(\mathbf{p}, q) \in \mathbb{Z}^{n+1}$ with $\left(\mathbf{p}^{\prime}, q\right)$ arbitrarily far from the origin such that

$$
\|\mathbf{p}+\mathfrak{a} q\| \leq \Pi_{+}\left(\mathbf{p}^{\prime}, q\right)^{-v / n} .
$$

Proof. This lemma is similar to Lemma 5.1 in [K11]; see also Case 1 of Theorem 4.5 in [G1]. Under the assumptions of the lemma, we take $0<\gamma<$ $\beta<1 / n$. Passing to a subsequence of the $t_{i}$ 's and reshuffling etc., we will assume that for some $k<n$,

$$
t_{i} \geq \gamma t \quad \text { for } i \leq k, \quad t_{i}<\gamma t \quad \text { for } i>k .
$$

This implies that if $i \leq k$,

$$
2^{-\beta t}\left|q_{i}\right|_{+} \leq 2^{t_{i}-\gamma t}
$$

and for $i>k$,

$$
2^{-\beta t}\left|q_{i}\right|<1 \quad \text { and } \quad 2^{-\beta t}|q|<1 .
$$

From (5.7) and (5.8), it follows that

$$
\Pi_{+}\left(\mathbf{p}^{\prime}, q\right) \leq \prod_{i=1}^{k}\left|p_{i}\right|_{+} \leq 2^{t[1+k(\beta-\gamma)]},
$$


which implies that for every $v>0$,

$$
\Pi_{+}\left(\mathbf{p}^{\prime}, q\right)^{-v / n} \geq 2^{-\frac{v}{n} t[1+k(\beta-\gamma)]} .
$$

Now define

$$
v=\frac{n(\gamma+1-\beta)}{1+k(\beta-\gamma)}
$$

Since we chose $0<\gamma<\beta$, we know that $v<n$. We thus get

$$
\|\mathbf{p}+\mathfrak{a} q\| \leq \Pi_{+}\left(\mathbf{p}^{\prime}, q\right)^{-v / n} .
$$

In view of Lemma 5.2 and the discussion preceding it, we get

Lemma 5.3. Assume that $\mathfrak{a}$ satisfies the conditions in Theorem 1.3 , Then for every $\beta>0$, there exists a positive $\gamma<\beta$ and $T=T(\beta)>0$ such that for every $\mathbf{t} \in \mathbb{Z}_{+}^{n}$ with $t \geq T(\beta)$,

$$
\sup _{\mathbf{x} \in B}\left\|\mathfrak{g}_{\beta, \mathbf{t}_{\mathbf{x}}} \Gamma\right\| \geq 2^{-\gamma t}
$$

for every $\Gamma \in \mathscr{P}(\Lambda)$ with $\operatorname{rank}(\Gamma)=1$.

5.2. Higher rank. In the intermediate cases, i.e. when $\Gamma$ has rank between 1 and $n$, our strategy will be to only consider terms which contain $\mathbf{e}_{0}$, as it is the expanding direction. Moreover, since our eventual aim is to bound $\left\|\mathfrak{g}_{\beta, \mathbf{t}} \mathfrak{u}_{\mathbf{x}} \mathbf{w}\right\|$ from below, it will be enough to restrict attention to those terms which further contain at most one $\mathbf{e}_{* i}$, so we can disregard those terms which contain expressions of the form $\mathbf{e}_{* i} \wedge \mathbf{e}_{* j}$. This suffices because an examination of the action of $\mathfrak{g}_{\beta, \mathbf{t}}$ reveals that terms which contain $\mathbf{e}_{* i} \wedge \mathbf{e}_{* j}$ have smaller norm than terms which contain at most one $\mathbf{e}_{* i}$. Thus the norm of $\left\|\mathfrak{g}_{\beta, t} \mathfrak{u}_{\mathbf{x}} \mathbf{w}\right\|$ is not realized on terms containing $\mathbf{e}_{* i} \wedge \mathbf{e}_{* j}$. In other words, we will really be computing the norm of a projection with kernel $\bigwedge^{2}\left(V^{*}\right)$ but we will suppress this in our calculations.

5.3. Example. Before we begin, we illustrate the computations involved with a simple example. Let us take the case of the line $(x, a)$, i.e. the hyperplane parametrized by the vector $\mathfrak{a}=(a, 0)$, define $\mathfrak{u}_{\mathbf{x}}, V, V^{\prime}$ as above and take $\mathbf{w} \in \bigwedge^{2}\left(\mathbb{R}^{3}\right)$ of the form

$$
\mathbf{w}=w \mathbf{e}_{0} \wedge \mathbf{e}_{2} .
$$

Then $\mathfrak{u}_{\mathbf{x}} \mathbf{w}=w \mathbf{e}_{0} \wedge\left(a \mathbf{e}_{0}+\mathbf{e}_{2}\right)=\mathbf{w}$.

Our objective would be to show that $\sup _{\mathbf{x} \in B}\left\|\mathfrak{g}_{\beta, \mathbf{t}} \mathfrak{u}_{\mathbf{x}} \mathbf{w}\right\|$ is not too small for all but finitely many $\mathbf{t}$. However, in this particular case the choice of $\mathbf{t}=(0, t)$ gives us that $\left\|\mathfrak{g}_{\beta, \mathbf{t}} \mathfrak{u}_{\mathbf{x}} \mathbf{w}\right\| \leq 2^{-2 \beta t}$ so we see that the condition $a_{i} \neq 0$ is essential to our approach. In fact, it has been observed by D. Kleinbock (personal communication, this can be adapted from Corollary 5.7 in [K11]) that $\mathfrak{a}=(a, 0)$ satisfies 5.5 if and only if $\omega(\mathfrak{a})<1$ where $\omega(\mathfrak{a})$ is the 
Diophantine exponent of $\mathfrak{a}$ defined as

$$
\omega(\mathfrak{a}):=\sup \left\{v: \mathfrak{a} \in \mathcal{W}_{v}(n, 1)\right\},
$$

which is known to be greater than or equal to 1 . Consequently, the condition imposed in Theorem 1.3 is never satisfied in this case, and a separate investigation is necessary. This study is carried out in a forthcoming work using quantitative nondivergence estimates developed in [K12].

For the purposes of this section, it will be convenient to use the following notation. For $1 \leq i<n$, we set $f_{i}=x_{i}$ and $f_{n}=\mathfrak{a} \tilde{\mathbf{x}}$. We also set $\mathbf{f}_{K}=$ $\left(f_{r}, f_{s}\right), K=\{r, s\}$ and $\mathbf{f}_{K}^{i}:=f_{r} \frac{\partial f_{s}}{\partial x_{i}}-f_{s} \frac{\partial f_{r}}{\partial x_{i}}$. Recall that for a vector $\mathbf{t} \in \mathbb{Z}_{+}^{n}$, we denote by $t$ the sum of its components. We will extend this notation as follows. For $J \subset\{1, \ldots, n\}$, we will set $t_{J}:=\sum_{j \in J} t_{j}$. We now take a nonzero vector $\mathbf{w} \in \bigwedge^{k}\left(V^{\prime}\right)$,

$$
\mathbf{w}=\sum_{I} w_{I} \mathbf{e}_{I}
$$

Here $I \subset\{0,1, \ldots, n\}, \# I=k$, and at least one coefficient $w_{I}$ is nonzero. When $\mathfrak{g}_{\beta, \mathbf{t}} \mathfrak{u}_{\mathbf{x}}$ acts on $\mathbf{w}$, the coefficient of $\mathbf{e}_{0} \wedge \mathbf{e}_{J}$ with $J \subset\{0,1, \ldots, n\}$, $\# J=k-1,0 \notin J$ is

$$
2^{-k \beta t} 2^{t-t_{J}}\left(w_{0 \cup J}+\sum_{j \notin J, j \neq 0} w_{j \cup J} f_{j}\right)
$$

while that of $\mathbf{e}_{0} \wedge \mathbf{e}_{* i} \wedge \mathbf{e}_{J}$ with $\# J=k-2$ is

$$
2^{-k \beta t} 2^{t-t_{J}}\left(\sum_{j \notin J, j \neq 0} w_{0 \cup j \cup J} \frac{\partial f_{j}}{\partial x_{i}}+\sum_{\substack{\# K=2 \\ K \cap J \neq \emptyset, 0 \notin K}} w_{J \cup K} \mathbf{f}_{K}^{i}\right) .
$$

We will use the following elementary observation which follows from the linear independence of the $f_{i}$ 's for $1 \leq i \leq n-1$ over $\mathscr{B}$ : for any subset $J$ of $\{0,1, \ldots, n\}$ with $\# J=k, n \notin J$, and for any nonzero $\mathbf{c} \in \mathbb{R}^{k+1}$ with $\|\mathbf{c}\| \geq 1$

$$
\sup _{\mathbf{x} \in B}\left\|c_{0}+\sum_{j \in J} c_{j} f_{j}\right\| \gg 1
$$

here and in the rest of the section, $\gg$ will be taken to mean $\geq$ a positive constant depending on $\mathscr{B}$ and/or $\mathfrak{a}$. In particular, this bounds the terms in the parentheses in (5.13) and (5.14) by a constant as above as long as $f_{n}$ is not present.

Now it can be assumed that at least one component of the vector $\mathbf{t}$, say $t_{i}$, is not very small, say $\|\mathbf{t}\|=t_{i} \geq t / n$. If $1 \leq i \leq n-1$, we can reorder the $t_{i}$ 's if necessary to assume that $t_{1} \geq t / n$. On the other hand, the linear dependence in the last coordinate means that we must separately consider 
the case when $t_{n} \geq t / n$. We first assume that

$$
t_{1} \geq t / n \text {. }
$$

The elementary but key observation we will use is that for any nonempty $J \subset\{1, \ldots, n\}$ with $1 \notin J$,

$$
2^{t-t_{J}} \geq 2^{t / n}
$$

In $\mathfrak{u}_{\mathbf{x}} \mathbf{w}$, we can clearly always find $\mathbf{e}_{K}$ with $1 \notin K$.

5.4. Case A. We assume first that

$$
\mathbf{e}_{K} \text { as above is of the form } \mathbf{e}_{0} \wedge \mathbf{e}_{J} \text {. }
$$

Then either $J$ contains $n$ or not. Assume first that $n \in J$. Then the term

$$
w_{0 \cup J}+\sum_{j \notin J, j \neq 0} w_{j \cup J} f_{j},
$$

which accompanies $\mathbf{e}_{0} \wedge \mathbf{e}_{J}$, does not contain $f_{n}$ and so in view of 5.16 (since $1 \notin J$ ) and (5.15),

$$
\sup _{\mathbf{x} \in B}\left\|\mathfrak{g}_{\beta, \mathbf{t}} \mathfrak{u}_{\mathbf{x}} \mathbf{w}\right\| \gg 2^{(-k \beta+1 / n) t} .
$$

If we cannot find a $J$ such that $n \notin J$, then in $\mathbf{w}=\sum w_{K} \mathbf{e}_{K}$, any $w_{K}$ such that $1 \notin K$ and $n \in K$ is zero. We now look for $J$ with $\# J=k-1$ such that $n \notin J$. Then $\mathbf{e}_{K}=\mathbf{e}_{0} \wedge \mathbf{e}_{J}$ can be written as

$$
w_{0 \cup J}+\sum_{j \notin J, j \neq 0, n} w_{j \cup J} f_{j}+w_{J \cup n} f_{n} .
$$

By assumption, $w_{J \cup n}=0$. If one of the other integers $w_{*}$ is nonzero, we can apply (5.18) once more.

5.5. Case B. Suppose Case $A$ is not satisfied, i.e. in $\mathfrak{u}_{\mathbf{x}} \mathbf{w}$, there is no $\mathbf{e}_{0} \wedge \mathbf{e}_{J}$ with $1 \notin J$. We can then find

$$
\mathbf{e}_{K}=\mathbf{e}_{0} \wedge \mathbf{e}_{* i} \wedge \mathbf{e}_{J}
$$

with $1 \notin J$. Recall that the coefficient of the above term is of the form 5.14). We now proceed exactly as before, namely we start with the assumption that $n \in J$. Since $n \in J, f_{n}$ does not appear in the above expression. Therefore, we can use (5.15) to get

$$
\sup _{\mathbf{x} \in B}\left\|\mathfrak{g}_{\beta, \mathbf{t}} \mathfrak{u}_{\mathbf{x}} \mathbf{w}\right\| \gg 2^{-k \beta t} 2^{t-t_{J}}
$$

In view of 5.16 , we have

$$
\sup _{\mathbf{x} \in B}\left\|\mathfrak{g}_{\beta, \mathbf{t}} \mathfrak{u}_{\mathbf{x}} \mathbf{w}\right\| \gg 2^{(-k \beta+1 / n) t}
$$

We treat the situation when $n \notin J$ in exactly the same fashion as in Case A and arrive at 
Lemma 5.4. For every $\beta>0$, every $\mathbf{t} \in \mathbb{Z}_{+}^{n}$ with $t_{1} \geq t / n$, and every $\Gamma \in \mathscr{P}(\Lambda)$ with $1<\operatorname{rank}(\Gamma)<n+1$,

$$
\sup _{\mathbf{x} \in B}\|H(\mathbf{x}) \Gamma\| \gg 2^{(-k \beta+1 / n) t} .
$$

We now consider the case when

$$
t_{n} \geq t / n \text {. }
$$

Similar to 5.16 we find that for any nonempty $J \subset\{1, \ldots, n\}$ with $n \notin J$,

$$
2^{t-t_{J}} \geq 2^{t / n}
$$

We will proceed in a manner similar to the proof of Lemma 5.4. As before, we can always find $\mathbf{e}_{K}$ with $n \notin K$.

5.6. Case A redux. We assume first that

$$
\mathbf{e}_{K} \text { as above is of the form } \mathbf{e}_{0} \wedge \mathbf{e}_{J}
$$

with $n \notin J$. The coefficient of such a term can be written as

$$
w_{0 \cup J}+\sum_{j \neq 0, n ; j \notin J} w_{j \cup J} f_{j}+w_{J \cup n} f_{n},
$$

or

$$
\left(w_{0 \cup J}+w_{J \cup n} a_{0}\right)+\sum_{j \neq 0, n ; j \notin J}\left(w_{j \cup J}+a_{j} w_{J \cup n}\right) f_{j}+w_{J \cup n} \sum_{j \in J} a_{j} f_{j} .
$$

Note that $f_{n}$ does not appear in the above list and so the functions above are linearly independent over $\mathscr{B}$. The only problem is that the terms $w_{j \cup J}+$ $a_{j} w_{J \cup n}$ need not be integers and so could be small. We will deal with this situation in the following manner. If $w_{J \cup n}=0$, then we can use (5.24) along with (5.15) to get

$$
\sup _{\mathbf{t} \in B}\left\|\mathfrak{g}_{\beta, \mathbf{t}} \mathfrak{u}_{\mathbf{x}} \mathbf{w}\right\| \gg 2^{(-k \beta+1 / n) t} .
$$

On the other hand, if $w_{J \cup n}$ is nonzero, we will focus attention on the term $w_{J \cup n} \sum_{j \in J} a_{j} f_{j}$. Since the $a_{j}$ 's are all nonzero by assumption, this term is nonzero. Moreover, since $\left|w_{J \cup n} a_{j}\right| \geq\left|a_{j}\right|$ for each $j$, we get

$$
w_{J \cup n} \sum_{j \in J} a_{j} f_{j} \gg \# J \min _{j \in J}\left|a_{j}\right| \gg \min _{1 \leq j \leq n}\left|a_{j}\right| .
$$

Thus, we can use (5.15) and (5.24) once more to get the bound (5.27).

On the other hand, if there are no terms of the form $\mathbf{e}_{0} \wedge \mathbf{e}_{J}$ with $n \notin J$, i.e. we can assume that all the integers appearing in (5.26) are zero, we then consider 
5.7. Case $\mathbf{B}$ redux. We assume $\mathfrak{u}_{\mathbf{x}} \mathbf{w}$ contains a vector $\mathbf{e}_{K}$ of the form $\mathbf{e}_{0} \wedge \mathbf{e}_{* i} \wedge \mathbf{e}_{J}$ with $n \notin K$. The coefficient of such a term can be written as

$$
\begin{aligned}
& \sum_{j \notin J, j \neq 0, n} w_{0 \cup j \cup J} \frac{\partial f_{j}}{\partial x_{i}}+w_{0 \cup J \cup n} \frac{\partial f_{n}}{\partial x_{i}} \\
&+\sum_{\substack{\# K=2, K \cap J=\emptyset \\
0, n \notin K}} w_{K \cup J} f_{K}^{i}+\sum_{\substack{\# K=2, K \cap J=\emptyset \\
0 \notin K, n \in K}} w_{K \cup J} f_{K}^{i} .
\end{aligned}
$$

By assumption, the integers $w_{0 \cup j \cup J}$ and $w_{K \cup J}$ above are zero. Thus the only nonzero terms are

$$
w_{0 \cup J \cup n} \frac{\partial f_{n}}{\partial x_{i}}=w_{0 \cup J \cup n}\left(a_{1} \frac{\partial f_{1}}{\partial x_{i}}+\cdots+a_{n-1} \frac{\partial f_{n-1}}{\partial x_{i}}\right) .
$$

In view of 5.15, we can conclude that for some $i$,

$$
\left\|w_{0 \cup J \cup n} \frac{\partial f_{n}}{\partial x_{i}}\right\| \gg 1
$$

which implies that

$$
\sup _{\mathbf{x} \in B}\left\|\mathfrak{g}_{\beta, \mathbf{t}} \mathfrak{u}_{\mathbf{x}} \mathbf{w}\right\| \gg 2^{-k \beta t} 2^{-t_{n} / 2} 2^{t-t_{J}} .
$$

Thus we can use (5.24) to get

$$
\sup _{\mathbf{t} \in B}\left\|\mathfrak{g}_{\beta, \mathbf{t}} \mathfrak{u}_{\mathbf{x}} \mathbf{w}\right\| \gg 2^{t[-k \beta+1 / n]} .
$$

We have thus proved, analogous to Lemma 5.4 .

Lemma 5.5. For every $\beta>0$, every $\mathbf{t} \in \mathbb{Z}_{+}^{n}$ with $t_{n} \geq t / n$, and every $\Gamma \in \mathscr{P}(\Lambda)$ with $1<r k(\Gamma)<n+1$,

$$
\sup _{\mathbf{x} \in \mathscr{B}}\|H(\mathbf{x}) \Gamma\| \gg 2^{(-k \beta+1 / n) t} .
$$

We now gather our calculations to prove Proposition 4.2. We take $\beta<$ $1 / n^{2}$ and using Lemmas 5.4 and 5.5 , we choose $T_{0}>0$ such that for every $\mathbf{w} \in \bigwedge^{k}\left(V^{\prime}\right)$ with $1<k \leq n+1\left({ }^{2}\right)$ and for every $\mathbf{t} \in \mathbb{Z}_{+}^{n}$ with $t \geq T_{0}$, we have

$$
\sup _{\mathbf{x} \in B}\left\|\mathfrak{g}_{\beta, \mathbf{t}} \mathfrak{u}_{\mathbf{x}} \mathbf{w}\right\| \geq 1
$$

For $\beta$ as above, we choose a $0<\gamma<\beta$ and $T(\beta)>0$ as in Lemma 5.3 . Take $T$ to be the bigger of $T_{0}$ and $T(\beta)$. It thus follows that for any $\Gamma \in \mathscr{P}(\Lambda)$, for any $\mathbf{t} \in \mathbb{Z}_{+}^{n}$ with $t \geq T$,

$$
\sup _{\mathbf{x} \in B}\|H(\mathbf{x}) \Gamma\| \geq 2^{-\gamma t},
$$

$\left({ }^{2}\right)$ For the case $k=n+1$, the analogues of Lemmas 5.4 and 5.5 are immediate. 
which establishes Proposition 4.2, thus completing the proof of Theorem 1.3 .

6. Weighted Diophantine approximation. We now turn to Theorem 1.2. The proof follows in a manner similar to Theorem 1.3 . We need to replace $(3.4)$ by

$$
\mathfrak{g}_{\beta, t, \mathbf{s}}=\operatorname{diag}\left(\frac{2^{-\beta t}}{2^{-n t / s}}, 2^{-\beta t}, \ldots, 2^{-\beta t}, \frac{2^{-\beta t}}{2^{t / s_{1}}}, \ldots, \frac{2^{-\beta t}}{2^{t / s_{n}}}\right),
$$

where $s=\sum_{i=1}^{n} s_{i}$. It is straightforward to see that a version of Lemma 5.3 can be obtained using (1.10). For the higher rank cases, we no longer have to consider the separate cases and can use Lemma 5.4 to obtain an analogue of Proposition 4.2 .

Acknowledgements. We thank the referee for several helpful suggestions and gratefully acknowledge support from NSF and EPSRC.

\section{References}

[Bu] Y. Bugeaud, Multiplicative Diophantine approximation, preprint.

[BBDD] V. Beresnevich, V. Bernik, H. Dickinson and M. M. Dodson, On linear manifolds for which the Khinchin approximation theorem holds, Vestsi Akad. Navuk Belarusi Ser. Fiz.-Mat. Navuk 2000, 14-17 (in Belorussian).

[BBKM] V. Beresnevich, V. Bernik, D. Kleinbock and G. Margulis, Metric Diophantine approximation: the Khintchine-Groshev theorem for non-degenerate manifolds, Moscow Math. J. 2 (2002), 203-225.

[BKM] V. Bernik, D. Kleinbock and G. A. Margulis, Khintchine type theorems on manifolds: the convergence case for the standard and multiplicative versions, Int. Math. Res. Notices 2001, no. 9, 453-486.

[D] M. M. Dodson, Geometrical and probabilistic ideas in metric Diophantine approximation, Uspekhi Mat. Nauk 48 (1993), 77-106 (in Russian); English transl.: Russian Math. Surveys 48 (1993), 72-103.

[Gal] P. Gallagher, Metric simultaneous Diophantine approximation, J. London Math. Soc. 37 (1962), 387-390.

[G1] A. Ghosh, A Khintchine-type theorem for hyperplanes, ibid. 72 (2005), 293-304.

[G2] -, A Khintchine-Groshev theorem for affine hyperplanes, preprint.

[G3] - Diophantine exponents and the Khintchine-Groshev theorem, preprint.

[Gr] A. V. Groshev, Un théorème sur les systèmes des formes linéaires, Dokl. Akad. Nauk SSSR 9 (1938), 151-152.

[K] A. Khintchine, Einige Sätze über Kettenbrüche, mit Anwendungen auf die Theorie der Diophantischen Approximationen, Math. Ann. 92 (1924), 115-125.

[K11] D. Kleinbock, Extremal subspaces and their submanifolds, Geom. Funct. Anal. 13 (2003), 437-466.

[Kl2] - An extension of quantitative nondivergence and applications to Diophantine exponents, Trans. Amer. Math. Soc. 360 (2008), 6497-6523.

[KM] D. Kleinbock and G. A. Margulis, Flows on homogeneous spaces and Diophantine approximation on manifolds, Ann. of Math. 148 (1998), 339-360. 
[Sp] V. G. Sprindžuk, Achievements and problems in Diophantine approximation theory, Russian Math. Surveys 35 (1980), 1-80.

Anish Ghosh

School of Mathematics

University of East Anglia

Norwich, UK NR4 7TJ

E-mail: a.ghosh@uea.ac.uk

Received on 6.8.2009

and in revised form on 12.12.2009 